\title{
Antecipação e processamento linguístico: uma revisão das principais evidências a favor da previsão
}

\author{
Anticipation and language processing: reviewing main evidences for prediction \\ mechanisms
}

\section{Mahayana Cristina Godoy}

Universidade Federal do Rio Grande do Norte - UFRN - Natal - Rio Grande do Norte - Brasil

\section{Cláudia Brandão Vieira}

\section{Maria Luiza Cunha Lima}

Universidade Federal de Minas Gerais - UFMG - Belo Horizonte - Minas Gerais - Brasil

Resumo: O presente artigo tem como objetivo discutir algumas das principais questões envolvidas na investigação das relações entre mecanismos antecipatórios e processamento da linguagem. Através da revisão da literatura das últimas duas décadas, apresentamos evidência de que o processamento linguístico é, por vezes, guiado por processos de antecipação, e que uma proposta meramente integrativa não é capaz de explicar os dados apresentados. Embora não tratemos de quão central são os processos antecipatórios para a compreensão da linguagem, argumentamos que o estudo de um potencial elétrico relacionado a eventos linguísticos - uma positividade frontal tardia - pode ajudar a responder uma série de questões que envolvem como, quando e o que se antecipa durante o processamento linguístico.

Palavras-chave: Antecipação. Processamento linguístico. Potenciais evocados.

Abstract: In this paper, some of the key issues involved in the the relationship between anticipatory mechanisms and language processing are discussed. By reviewing studies from the last two decades, we present evidence that linguistic processing is sometimes guided by anticipation mechanisms and, therefore, a solely integrative model cannot account for the presented data. Although we do not discuss how central these anticipatory processes are for language comprehension, we argue that the study of an event-related potential - a late frontal positivity - can be helpful in answering questions such as how, when and what we predict during language processing.

Keywords: Anticipation. Language processing. Event-related potentials. 


\section{Introdução}

A ideia de que a antecipação seria um componente importante da arquitetura do processamento linguístico provém da noção intuitiva de que, frente a contextos altamente restritivos como na sentença "após ouvir os parabéns, o aniversariante assoprou as...", nosso processador seria capaz de antecipar um item lexical específico - no caso, "vela" ou, ao menos, alguns de seus traços lexicais. Apesar de intuitiva, a noção de que o processamento linguístico seria guiado por processos de antecipação não encontrou, historicamente, espaço entre os modelos que pretendiam explicar a compreensão, a produção e a aquisição da linguagem. Como apontam DeLong et al. (2014), o motivo possivelmente reside na imensidão de combinações linguísticas, que fariam da antecipação de um item lexical específico um processo muito custoso (JACKENDOFF, 2002), na afluência de modelos modulares de processamento (e.g., FODOR, 1983; FORSTER, 1989) e no uso de métodos experimentais offline que não conferem uma boa resolução temporal.

No entanto, as evidências experimentais que se acumulam na última década tornaram datados os questionamentos quanto à existência ou não de mecanismos antecipatórios e levaram grupos de pesquisadores a se convencerem de que as perguntas mais urgentes deveriam ser quando, como e por que a antecipação ocorre (VAN PETTEN e LUKA, 2012). As edições especiais dedicadas à relação entre processamento linguístico e antecipação em números recentes das revistas Cortex ${ }^{1}$ e Language, Cognition and Neuroscience ${ }^{2}$ são apenas um dos exemplos de como a antecipação passou a constituir, nos últimos anos, um campo de estudo per se na psicolinguística, instigando pesquisas que se ocupam primordialmente em defini-la conceitualmente, explicar seus limites e meios de atuação e seu papel na compreensão, aquisição e produção da linguagem.

$\mathrm{Na}$ esteira desse interesse crescente, pesquisadores da comunidade internacional têm dado

\footnotetext{
1 Cortex. Special Issue: Prediction in speech and language processing. v. 68, 182 p., 2015.
}

à antecipação papel de destaque no estudo de fenômenos como processamento sintático (FINE e JAEGER, 2013), o processamento do sinal acústico (ASTHEIMER e SANDERS, 2011; BROWN et al., 2011; SALVERDA et al., 2014), a elaboração das relações de coesão e coerência (ROHDE e HORTON, 2014; KEHLER e ROHDE, 2013) ou a interação entre processamento linguístico e diferenças individuais (WLOTKO et al., 2012; HUETTIG e JANSE, 2016). Apesar do intenso debate no cenário internacional, não há publicações sobre o tema no Brasil (à exceção da dissertação de Vieira (2015)).

O presente artigo tem como objetivo preencher essa lacuna, apresentando, em português, uma resenha dos pontos principais envolvidos no estudo dos processos antecipatórios. Nossa intenção é trazer o debate para o plano nacional e estimular pesquisas na área que possam acompanhar as tendências internacionais sobre o tema. Para tanto, dividiremos nosso texto em duas frentes. Primeiramente, apresentamos as discussões iniciais (e que consideramos superadas) sobre a existência ou não de mecanismos de antecipação, travadas principalmente na década passada. Na sequência, tratamos de como a investigação experimental sobre o assunto vêm sendo conduzido. Embora os estudos sejam feitos a partir das mais diversas técnicas, o que inclui leitura auto-cadenciada (FINE e JAEGER, 2013), rastreamento ocular (KIM et al., 2015), eletroencefalografia (LASZLO e FEDERMEIER, 2009; WLOTKO e FEDERMEIER, 2015), neuroimagem (DIKKER et al., 2014; DIKKER e PYLKKÄNEN, 2013) e modelamento computacional (SMITH e LEVY, 2013), trataremos do tema a partir de uma revisão dos potenciais evocados por eletroencefalografia durante 0 processamento da linguagem.

\section{Antecipação ou facilidade de integração: duas explicações alternativas para os mesmos dados}

Nas últimas décadas, estudos sobre a influência que informações pragmáticas exercem no

2 Language, Cognition and Neuroscience. Special Issue: Prediction in Language. v. 31, n. 1, 165 p., 2016. 
processamento linguístico estão entre os que mais contribuíram para o consenso de que o processador age, pelo menos por vezes, de modo antecipatório. Embora hoje seja menos controverso assumir a existência de mecanismos de antecipação, inicialmente a ideia era questionada devido à possibilidade de uma hipótese alternativa: a facilidade de integração. Segundo essa hipótese, o processador não anteciparia informações linguísticas, mas as integraria com mais facilidade a depender do contexto sentencial ou discursivo prévio. Como veremos nessa seção, a facilidade de integração se colocou como hipótese alternativa devido, principalmente, aos limites das metodologias empregadas para investigar a questão.

Um dos paradigmas mais utilizados para investigar processos de antecipação, pelo menos em um primeiro momento, foi o paradigma do mundo visual. Nessa técnica experimental, frases são apresentadas auditivamente aos participantes enquanto um rastreador ocular registra, com precisão, para onde o sujeito está olhando enquanto ouve o estímulo linguístico. Altman e Kamide (1999) identificaram que, ao ouvir uma frase como o menino vai comer..., os participantes olhavam para a figura de um bolo (único referente comestível) apresentada na tela do computador, ignorando outras figuras que também estavam presentes - uma bola, um carrinho e um trem de brinquedo. Esses resultados mostram, segundo os autores, que restrições impostas por traços lexicais do verbo são capazes de guiar a atenção (e o olhar) dos participantes para referentes que têm mais chances de serem mencionados. Como o olhar é direcionado antes da apresentação auditiva do referente, ainda no momento em que o sujeito está processando o verbo, assume-se que o processador age de modo a antecipar referentes possíveis.

Embora esse estudo foque em restrições lexicais impostas pelo verbo, outros experimentos indicam que o conhecimento de mundo sobre eventos, algo que emergiria da interação entre argumento externo e verbo, também influencia processos de antecipação de referentes. Em outro estudo que fez uso do paradigma mundo visual, os participantes viam uma cena com dois possíveis agentes e quatro objetos (KAMIDE et al., 2003). Em um dos cenários apresentados no estudo, havia uma menina e um homem (agentes) e figuras de um copo de cerveja, uma motocicleta, um carrossel e um doce. Os participantes se detiveram mais sobre a figura de uma motocicleta durante o processamento do verbo na frase o homem vai montar... do que quando ouviam a menina vai montar..., o que levou os autores a concluírem que o conhecimento de eventos dos sujeitos impactava seus movimentos oculares, que, por sua vez, mostravam um processo de antecipação do argumento interno da oração.

Outros estudos, dessa vez fazendo uso de técnicas de eletroencefalografia, também encontraram efeitos de que conhecimento pragmático impactaria 0 processamento de sentenças. Manipulando as expectativas que os sujeitos construíam para o trecho final de uma sentença (e.g., Eles queriam deixar o hotel com cara de um resort tropical. Então, ao longo do caminho, plantaram...), Federmeier e Kutas (1999) puderam observar os custos envolvidos no processamento de uma palavra esperada (e.g., palmeiras) comparativamente ao processamento de palavras implausíveis com o contexto discursivo, mas que, ou estavam na mesma categoria do final esperado (e.g., pinheiros), ou faziam parte de outra categoria (e.g., tulipa). Os resultados indicaram facilitação do processamento de palavras esperadas, e as autoras concluíram que "o contexto leva a antecipações ou expectativas específicas" (p. 484), e que "ao longo do curso do processamento de uma sentença, o sistema de compreensão está envolvido com algum processo equivalente a antecipação" ( $p$. 487).

Estudos como os citados fomentaram a discussão acerca da existência de mecanismos antecipatórios, mas ainda não conseguiam separar o efeito de antecipação de um possível efeito de facilitação de integração. No caso dos experimentos que usam o paradigma do mundo visual, argumentase não haver evidência de processos de antecipação, pois uma explicação alternativa seria de que o sujeito seleciona, entre os referentes já apresentados na tela 
do computador, aquele que completaria melhor a sentença. Embora o sintagma o bolo ou a motocicleta não tivessem sido apresentados de forma explícita, os referentes já estavam dados visualmente aos voluntários da pesquisa, o que enfraquece 0 argumento de que a seleção do argumento interno se deu apenas por mecanismos antecipatórios acionados pelas restrições impostas pelo verbo.

Quanto a experimentos como os de Federmeier e Kutas (1999), uma explicação alternativa seria de que o processador integra com mais facilidade as palavras relacionadas ao contexto por causa de ativações feitas a partir dos itens lexicais e da imagem construída ao longo do discurso. Nesse caso, não haveria pré-ativação de traços de palmeiras por causa da expectativa gerada pelos leitores. Embora as autoras argumentem contra essa explicação, que se baseia mais em uma facilidade de integração do que em antecipação propriamente dita, o fato de seu segmento crítico de análise ser justamente a palavra (supostamente) antecipada não permite investigar se, antes da apresentação da palavra, alguns de seus traços já estavam antecipados.

Em todos esses trabalhos citados, não há, portanto, evidências empíricas que não possam ser explicadas por uma hipótese alternativa: a de que o processador não antecipa informações linguísticas, mas apenas as integra com mais facilidade a depender de um contexto prévio. Além disso, mesmo que aceitemos a hipótese de antecipação com esses dados, ainda se deve responder o que, exatamente, o processador antecipa. Seria possível realmente antecipar um item lexical específico, como bolo ou palmeiras, ou há, na verdade, antecipação de traços de um campo semântico mais amplo? No caso de haver antecipação de um item lexical, antecipa-se apenas seus traços semânticos ou também informações de conhecimento de mundo associadas a ele? Por fim, havendo a antecipação do item lexical, haveria também a antecipação de suas características fonológicas, morfológicas e sintáticas?
Respostas a essas perguntas começaram a ser esboçadas a partir do uso de outros paradigmas experimentais que investigavam o processamento de palavras antes da apresentação do item que confirmaria ou violaria as expectativas dos ouvintes/falantes. Nesse ponto, é importante ressaltar a importância do estudo dos potenciais relacionados a eventos $^{3}$ (ERPs, na sigla em inglês) como forma de compreender melhor os mecanismos que guiam os processos de antecipação. Na próxima seção, nos dedicamos a alguns dos pontos principais associados ao uso de ERPs nas perquisas sobre antecipação.

\section{N400 e antecipação}

O componente N400 é uma onda cerebral de polaridade negativa cujo pico acontece em torno de 400ms após a apresentação de um estímulo. Esse componente geralmente é mais bem identificado nas regiões pariental e central do cérebro, com amplitudes ligeiramente maiores no hemisfério direito.

$\mathrm{Na}$ década de 80, o N400 tornou-se conhecido na literatura psicolinguística como um índice de facilidade de acesso a informações de significado. Em geral, todas as palavras de significado produzem o N400. Sabe-se que a amplitude desse componente é sensível a fatores relacionados à facilidade de acesso a informações na memória - como frequência da palavra, repetição, vizinhança ortográfica (KUTAS et al., 2006).

Além disso, existem fortes evidências (KUTAS e HILYARD, 1980; 1984) de que a amplitude do componente também é influenciada - de modo inversamente proporcional - pela expectativa de ocorrência de uma palavra em um determinado contexto discursivo. Kutas e Hillyard (1980) mostraram que durante a leitura de frases como Eu prefiro café com creme e... palavras conceitualmente esperadas nesse contexto, como a palavra açúcar, provocavam a diminuição da amplitude do N400 se comparadas a palavras conceitualmente inesperadas, como a palavra meias.

\footnotetext{
${ }^{3}$ Para uma descrição detalhada das caraterísticas dos potenciais evocados, ver Luck (2014).
} 
Em geral, a expectativa pela presença de uma palavra é medida por meio de probabilidades de cloze (TAYLOR, 1953). Ao utilizar tarefas de continuação de sentenças, esse método quantifica a possibilidade de ocorrência de certas palavras de acordo com os contextos sentenciais ou discursivos que as antecedem. A amplitude do N400 é fortemente relacionada à probabilidade de cloze de uma palavra (KUTAS e HILYARD, 1984; VAN PETTEN e KUTAS, 1990). Quando comparadas a palavras de alta probabilidade de cloze (probabilidade média de 0.93), palavras de baixa probabilidade (probabilidade média de 0.29) apresentaram um N400 de maior amplitude (KUTAS e HILYARD, 1984). Entretanto, palavras de baixa probabilidade (probabilidade abaixo de 0.05 ) também podem apresentar certa redução do N400, caso compartilhem traços semânticos com palavras de alta probabilidade (FEDERMEIER e KUTAS, 1999).

Há certa dificuldade em se definir o tipo de processo responsável por desencadear a redução da amplitude do N400. É possível que a redução da amplitude do componente seja motivada por processos de antecipação, nos quais os traços conceituais de uma palavra encontram-se ativos antes de sua leitura ou audição. Contudo, há possibilidade da redução do $\mathrm{N} 400$ também refletir a facilidade de se integrar traços conceituais à representação discursiva. (Cf.: HAGOORT, 2007). Portanto, para que seja possível a dissociação desses dois processos, é necessário que o experimento de potenciais evocados possua um desenho capaz de identificar o efeito de facilitação em um momento anterior à apresentação da palavra alvo.

Em um experimento de leitura de frases, DeLong e colegas (2005) encontraram evidências de que processos de antecipação influenciam a amplitude do N400. No experimento, os pesquisadores usaram manipulações que exploravam o conhecimento de mundo dos leitores e a variação entre os artigos indefinidos $a$ e an em inglês. Embora possuam significados idênticos, esses artigos têm a ocorrência direcionada pelo fonema inicial da palavra seguinte. Os pesquisadores criaram frases que poderiam ser completadas por palavras esperadas ou palavras inesperadas, porém plausíveis. O artigo adequado para a palavra esperada era sempre diferente daquele adequado para a palavra inesperada. No experimento, as palavras da frase eram apresentadas uma a uma, de maneira que os participantes não fossem capazes de ler o artigo juntamente com a palavra alvo. A seguir, apresentamos um exemplo das frases utilizadas:

(1) The day was breezy so the boy went outside to fly* ...

* Ventava naquele dia, então o menino saiu para empinar

(2)... a kite in the park* (Continuação com palavra esperada)

* uma pipa no parque

(3)... an airplane in the park ${ }^{*}$ (Continuação com palavra inesperada)

* um avião no parque

Os resultados mostraram que as amplitudes do N400 nos artigos a e an eram influenciadas pela expectativa por certas palavras. Artigos adequados para palavras esperadas (e.g., a para kite) apresentavam um $\mathrm{N} 400$ significantemente menor do que o N400 encontrado em artigos inadequados palavras esperadas, mas adequados para palavras inesperadas (e.g., an para airplane).

O estudo de DeLong et al. (2005) é bastante relevante para as propostas que relacionam a antecipação ao processamento da linguagem, pois mostra que os participantes foram capazes de prever a forma ortográfica dos sintagmas nominais mais esperados, antes mesmo de visualizá-los. Os resultados sugerem que os participantes utilizaram a representação contextual e conhecimento mais refinados sobre eventos do mundo real para prever ativamente que, na sentença em que se descreve um dia de muito vento, seria mais provável que o verbo fly fosse seguido por kite (pipa) do que por airplane (avião). Como as duas formas do artigo possuem significados idênticos, esses resultados não podem ser relacionados à facilidade/dificuldade de integrar informações conceituais, mas que os participantes anteciparam a forma ortográfica da palavra a ser apresentada. 
O trabalho de Delong et al. (2005) é um de uma série de estudos delineados com o objetivo de averiguar a existência de processos antecipatórios e questões como que tipo de informação pode fazer com que o processador antecipe informações linguísticas e que tipo de informações são antecipadas. Pesquisas em espanhol, holandês e polonês (WICHA et al., 2004; VAN BERKUM et al., 2005 e SZEWCZYK e SCHRIEFERS, 2013) também adotaram paradigma semelhante para investigar essas questões, apresentando, antes do nome supostamente antecipado, artigos ou adjetivos que violassem ou não as expectativas dos leitores/ouvintes (e.g., Caperucita Roja cargaba la comida para su abuela en una canasta (complemento esperado)/un sesto (complemento inesperado)). De modo geral, os autores reportam aumento de custos de processamento relacionados à leitura/audição de artigos ou adjetivos quando há violação de expectativas, indicando não apenas que a antecipação é parte dos mecanismos envolvidos no processamento linguístico, mas também que informações gramaticais de itens lexicais, como seu gênero, podem ser ativadas por pistas contextuais da sentença/discurso.

Embora a amplitude do N400 seja um bom parâmetro para mensurar os benefícios da confirmação de uma antecipação, há certa dificuldade em se identificar os custos provocados por qualquer falha no processo de antecipação. É possível que a amplitude do $\mathrm{N} 400$ reflita apenas os benefícios produzidos pela confirmação de uma de uma previsão. Ou seja, é possível que as diferenças entre as amplitudes do $\mathrm{N} 400$ de palavras esperadas e não esperadas reflitam apenas as facilidades de se acessar uma palavra cujos traços conceituais, as informações de gênero ou até mesmo a forma ortográfica já foram antecipados.

DeLong et al. (2011) sugerem que a amplitude do N400 é sensível às probabilidades de cloze, mas não a diferentes intensidades de restrições contextuais. Por exemplo, nas frases a seguir, a palavra fazenda é considerada uma continuação inesperada e possui probabilidades de cloze semelhantes (abaixo de 0.03):

(4) Sua pele estava vermelha por passar o dia na (fazenda).

(5) João construiu sua nova casa em uma silenciosa (fazenda).

No entanto, a frase (4) é considerada mais informativa, pois nela existe uma forte expectativa (cloze acima de 0.7) pelo aparecimento da palavra praia. Já a frase (5) não é considerada tão informativa, pois não há uma forte expectativa por nenhuma palavra específica. Diversas palavras foram citadas como possíveis continuações para essa frase (e.g., rua, região, vizinhança, montanha).

Federmeier et al. (2007) não encontraram diferenças significativas entre o $\mathrm{N} 400$ produzido pela palavra fazenda em frases semelhantes às citadas acima. Dito de outro modo, palavras inesperadas (com probabilidades de cloze abaixo de 0.03) que ocorrem após contextos muito informativos, nos quais os processos de antecipação parecem ser mais robustos, possuem o N400 semelhante ao verificado em palavras inesperadas que ocorrem após contextos pouco informativos, nos quais os processos de antecipação parecem ser mais leves. A partir desses dados, argumenta-se que, se houvesse de fato uma antecipação de informação linguística, deveria haver evidência empírica de que a quebra dessa expectativa é mais custosa quanto mais informativo for o contexto, o que não é o caso ${ }^{4}$.

A dificuldade em se estabelecer a forma como os custos de uma falha de antecipação podem influenciar componentes de ERP é tratada como evidência a favor dos modelos de compreensão mais focados em processos integrativos (SCHWANENFLUGEL e SHOBEN, 1985; TRAXLER e FOSS, 2000). Contudo, Kutas (1993) e Federmeir et al. (2007) encontraram evidências de que um componente de ERP, diferente do N400, pode ser sensível aos custos processuais gerados pela falta de convergência entre a informatividade do contexto e a

\footnotetext{
${ }^{4}$ Cf. DeLong et al. (2014) para uma linha de argumentação nesse sentido.
} 
previsibilidade de palavras. Tal componente é conhecido como positividade frontal tardia.

$\mathrm{Na}$ seção a seguir, apresentaremos as características e faremos uma breve revisão dos estudos indicam a relação desse componente com processos de antecipação.

\section{Positividade frontal tardia e antecipação}

Recentemente, uma positividade localizada na região frontal do cérebro com latência entre 500 e $900 \mathrm{~ms}$ passou a ser relacionada a falhas no processo de antecipações durante o processamento da linguagem (DELONG et al., 2014; DELONG et al. 2011; WLOTKO et al., 2012; THORNHILL e VAN PETTEN, 2012;). Essa relação se deve ao fato de, em grande parte dos estudos, o componente ser identificado apenas em situações em que o contexto era bastante informativo, com fortes expectativas por palavras específicas.

Uma das primeiras menções a esse tipo de positividade frontal foi realizada por Kutas (1993). No estudo, a pesquisadora relata que uma positividade com distribuição frontal e latência entre 500 e 900ms foi identificada após a leitura de palavras com baixas probabilidades de cloze em sentenças muito restritivas. Para a pesquisadora, essa positividade pode ser um índice do custo processual provocado pela não ocorrência de uma palavra muito esperada.

Federmeier et al. (2007) utilizaram diferentes níveis de restrições contextuais para tentar compreender os custos processuais provocados pela falha de uma previsão. Os pesquisadores exploram sentenças com restrições contextuais fortes e fracas para identificar os custos de processamento provocados por palavras com baixas probabilidades de cloze. Para excluir qualquer tipo de facilitação, foi realizado um controle para que as palavras inesperadas não possuíssem traços conceituais em comum com as palavras mais esperadas.

Os resultados indicaram o aparecimento de uma positividade frontal entre $500-900 m s$ após a leitura de palavras com baixas probabilidades de cloze. Porém, essa positividade foi identificada apenas em sentenças com fortes restrições contextuais. Os pesquisadores sugerem que processos de antecipação ocorrem consistentemente apenas durante a leitura de sentenças com fortes restrições contextuais - proposta que é refutada por modelos recentes que consideram a antecipação como uma atividade inerente ao processamento da linguagem (KUPERBERG e JAEGER (2015); KUPERBERG (2016)).

Outros estudos (DELONG et al., 2014; DELONG et al. 2011; WLOTKO, FEDERMEIER e KUTAS, 2012; THORNHILL e VAN PETTEN, 2012;) têm sido propostos a fim de melhor esclarecer as características desse componente. Em geral, os pesquisadores concordam que a positividade frontal tardia parece refletir as consequências de se préativar, mas não receber, informações conceituais altamente esperadas. Eles também distinguem esse componente do chamado P600 semântico, que possui distribuição parietal e está relacionado a violações de traços semânticas brutos, como papéis temáticos e animacidade.

A positividade frontal tardia parece estar relacionada à ocorrência de palavras inesperadas e plausíveis. Palavras inesperadas e plausíveis são aquelas que, apesar de possuírem uma probabilidade de cloze reduzida, podem perfeitamente ocorrer após certos contextos (e.g., a palavra laranja na frase: durante a festa, o churrasqueiro fatiou suculentos pedaços de laranja para os convidados). Ao reanalisar os resultados de DeLong et al. (2005) - citado na seção 3 - Delong et al. (2011) identificaram a ocorrência de uma positividade frontal e prolongada quando nomes inesperados e plausíveis eram lidos durante o experimento. Van Petten et al. (2012) revisaram os resultados de diversos estudos em que foram identificadas positividades tardias positividades que ocorrem após $400 \mathrm{~ms}$ do início da palavra. Os autores sugerem que a positividade normalmente relacionada a palavras inesperadas $\mathrm{e}$ implausíveis possui distribuição parietal, similar à distribuição do chamado P600 semântico, e, portanto, diferente da positividade frontal associado às palavras plausíveis. 
O estudo de Wlotko, Federmeier e Kutas (2012) indicou que a positividade frontal identificada por Federmeier et al. (2007) ocorre primordialmente em jovens adultos. Para esses pesquisadores, adultos mais velhos (com idade entre 59 e 88 anos) são menos propensos a utilizar estratégias de antecipação durante a compreensão da linguagem, quando comparados a jovens adultos (com idade entre 18 e 28 anos). É possível que essa diferença nas estratégias de compreensão seja motivada pela diminuição da eficiência com que adultos mais velhos utilizam informações contextuais para prever palavras que poderão ocorrer nas sentenças.

Há evidências de que a positividade frontal tardia, ao contrário do $\mathrm{N} 400$, não seja sensível à similaridade semântica entre palavras. Em Thornhill e Van Petten (2012), palavras imprevisíveis que possuíam traços conceituais relacionados às palavras mais esperada apresentaram uma positividade frontal pós $\mathrm{N} 400$ de amplitude semelhante à de palavras imprevisíveis que não possuíam traços conceituais relacionados às palavras mais esperada. Os pesquisadores interpretam esse resultado como uma evidência de que a positividade frontal reflete um nível de análise que está relacionado à antecipação de informações especificamente lexicais (e.g., forma ortográfica/ fonológica, categoria gramatical, frequência de uso) e não à antecipação de informações conceituais.

Por fim, diferente de Federmeier et al. (2007), os resultados de Thornhill e Van Petten (2012) mostraram que palavras inesperadas em contextos pouco restritivos também são capazes de produzir positividades frontais tardias. A motivação para a divergência de resultados ainda não foi claramente identificada. Embora os pesquisadores apontem que a utilização de testes de cloze cronometrados possa ter influenciado a natureza das sentenças com contextos pouco restritivos, é possível que outros fatores ainda desconhecidos possam ter influenciado o componente.

É importante destacar que os trabalhos que tratam da positividade frontal tardia são bastante cautelosos em suas conclusões e enfatizam a necessidade de se realizar investigações mais profundas. Diferentemente do N400, a respeito do qual há uma literatura bastante consistente, as propriedades da positividade frontal tardia ainda são pouco conhecidas. Portanto, diante do pequeno número de estudos realizados até o momento, fica clara a necessidade de se promover pesquisas mais consistentes sobre as caraterísticas do componente.

\section{Conclusão}

Este trabalho apresenta uma introdução aos principais pontos relacionados à ocorrência de processos antecipatórios durante o processamento da linguagem. Em nossa exposição mostramos que, ao longo das últimas duas décadas, diversas pesquisas (WICHA et al., 2003; 2004; DELONG et al., 2005; VAN BERKUN et al., 2005; FEDERMEIER et al., 2007; VAN PETTEN e LUKA, 2012) foram capazes de apresentar evidências bastante robustas de que a antecipação de informações é uma das estratégias utilizadas inconscientemente por falantes/ouvintes. Contudo, o corpo de evidências reunido até agora apenas descortina uma série de perguntas que devem ser investigadas nos próximos anos.

Uma dessas questões diz respeito ao papel da antecipação no processamento da linguagem: seria o de protagonista, elemento crucial para 0 processamento, ou de coadjuvante, apenas um dos mecanismos envolvidos em vários processos necessários para o processamento linguístico? Alguns autores defendem que a antecipação tem papel central em vários aspectos do processamento da linguagem, incluindo a aquisição/aprendizado de estruturas (SAFFRAN et al., 1996; CHANG et al., 2006) e a organização de turnos conversacionais (PICKERING e GARROD, 2013). Frequentemente, tais modelos também assumem que processos de antecipação afetam o processamento linguístico porque estariam no cerne dos processos cognitivos mais gerais (FARMER et al., 2013; CLARK, 2013). Outros tomam uma posição mais moderada acerca do papel da antecipação no processamento da linguagem e, embora reconheçam que tal mecanismo cumpra uma função importante, não o consideram peça central ou 
fundamental (HUETTIG e MANI, 2016; HUETTIG, 2015). Alguns pesquisadores que tomam essa posição assumem que o processamento da linguagem não pode ser explicado por meio de propostas estritamente antecipatórias ou exclusivamente integrativas, sugerindo que o processador, pelo menos na compreensão da linguagem, agiria a partir da combinação desses dois fatores (DELONG et al., 2014).

Alguns dos principais argumentos para uma posição mais conservadora sobre o papel da antecipação no processamento linguístico reside nas evidências de que alguns grupos de falantes/ouvintes teriam mais dificuldades para antecipar informações linguísticas de trechos subsequentes de um texto ou sentença. Tais evidências são encontradas em estudos que sugerem que adultos mais velhos fazem menos uso de processos antecipatórios comparativamente a jovens adultos (FEDERMEIER e KUTAS, 2005; FEDERMEIER et al., 2010), que maior proficiência de leitura correlaciona positivamente com o uso de mecanismos de antecipação (MISHRA et al., 2012; HUETTIG e BROUWER, 2015) e que falantes não nativos usam menos pistas discursivas para antecipar informações linguísticas (GRUTER et al., 2014). Alternativamente, pode-se argumentar que envelhecimento, falta de proficiência de leitura e uso de segunda língua revelam contextos em que o processamento da linguagem não opera em condições ótimas, o poderia ser, justamente, a consequência de menor capacidade de uso dos mecanismos antecipatórios.

Além da discussão acerca do papel que têm a antecipação no processamento linguístico, as questões suscitadas pelo estudo dos mecanismos antecipatórios resvalam em pontos que pautaram o estudo do processamento da linguagem nas últimas décadas. Nesse aspecto, reformulam-se velhas questões como a natureza da arquitetura do processamento linguístico, que pode ser definido como uma sucessão de estágios distintos (e.g., FRAZIER e FODOR, 1978), ou um mecanismo que agiria por restrições impostas por pistas lexicais, indicadores de frequência ou outras pistas contextuais (e.g., SPIVEY-
KNOWLTON e SEDIVY, 1995). Atualmente, essa discussão não é central para os estudos sobre antecipação, mas pode ser vista na disputa entre propostas que se colocam como herdeiras dos modelos baseados em restrições que ganharam terreno a partir da década de noventa (KUPERBERG, 2016) e explicações que postulam heurísticas distintas que agiriam serialmente, cada uma lidando com um tipo de informação, como processamento lexical, sintático ou atribuição de papeis temáticos ( $\mathrm{CHOW}$ et al., 2015; KUPERBERG, 2007).

Para além dessas questões mais gerais sobre a arquitetura do processamento linguístico e o papel que a antecipação desempenharia nesse construto, há uma gama de pontos mais específicos a serem ainda investigados. Nesse sentido, há ainda de se entender como age o mecanismo antecipatório no processamento da linguagem e que tipo de informação se antecipa ou se usa para antecipar. Com relação à pergunta sobre como a antecipação ocorre, uma das principais discussões centra-se nas possibilidades de os mecanismos subjacentes ao processo serem (a) semelhantes a um processo automático de priming ou (b) derivados de processos controlados (DeLong et al., 2014). No primeiro caso, o mecanismo antecipatório seria resultado de uma ativação de redes de associação passiva entre um item lexical que agiria como prime e seu alvo (e.g., médico $\rightarrow$ paciente); no segundo, o processo não se limitaria a uma relação entre palavras, mas seria derivado da construção de uma representação discursiva que restringiria fortemente as continuações possíveis para a sentença. Embora haja evidências favorecendo a segunda hipótese (Otten e Van Berkum, 2008; Van Berkum et al., 2005), trabalhos recentes argumentam que as duas estratégias são acionadas nos processos de antecipação (Hintz et al., 2015, Huettig, 2015).

Por fim, a discussão acerca do tipo de informação usada para engajar processos de antecipação tem sido central nos estudos sobre o tema, e levou a um consenso de que informações das mais diversas ordens impactam o processamento linguístico (cf. Huettig, 2015). Contudo, embora haja evidências de que informações de diferentes níveis 
(e.g., ortográficas, conceituais, morfológicas) são préativadas durante o processamento, pouco se sabe sobre os mecanismos responsáveis por desencadear e até mesmo redirecionar as informações antecipadas. Uma das questões que ainda se encontra em discussão diz respeito à identificação de processos inibitórios que deveriam ocorrer diante do aparecimento de informações completamente inesperadas, ou seja, diante da falha de uma previsão. Os estudos que sugerem a relação entre positividade frontal tardia e processos inibitórios (DELONG et al., 2014; DELONG et al. 2011; WLOTKO, FEDERMEIER e KUTAS, 2012; THORNHILL e VAN PETTEN, 2012) ainda são bastante incipientes, e, como o componente em questão apresenta latência tardia, é possível que seu comportamento reflita outros processos cognitivos. Por esse motivo - e em linha com o que argumentamos ao longo do presente artigo acreditamos que estudos mais detalhados sobre a natureza desse componente são necessários para lidar com questões sobre a natureza e funcionamento dos mecanismos de antecipação no processamento da linguagem.

\section{Referências}

ALTMANN, Gerry T. M.; KAMIDE, Yuki. Incremental interpretation at verbs: Restricting the domain of subsequent reference. Cognition, v. 3, n. 73, p. 247264, dez. 1999.

ASTHEIMER, Lori B.; SANDERS, Lisa D. Predictability affects early perceptual processing of word onsets in continuous speech. Neuropsychologia, v. 12, n. 49, p. 3512-3516, out. 2011.

BROWN, Meredith; SALVERDA, Anne Pier; DILLEY, Laura C.; TANENHAUS, Michael K. Expectations from preceding prosody influence segmentation in online sentence processing. Psychonomic Bulletin \& Review. v. 18, n. 6. p. 1189-1196. nov. 2011.

CHANG, Franklin; DELL, Gary S.; BOCK, J. Kathryn. Becoming syntactic. Psychological Review, v. 2, n. 113, p.234-272, abr. 2006.

CHOW, Wing Yee; SMITH, Cybelle; LAU, Ellen; PHILLIPS, Colin. A 'bag-of-arguments' mechanism for initial verb predictions. Language, Cognition, \& Neuroscience, n. 30, v. 9, p. 1-20, set. 2015.

CLARK, Andy. Whatever next? Predictive brains, situated agents, and the future of cognitive science.
Behavioral and Brain Sciences, v. 36, n. 03, p. 181204, jun. 2013.

DELONG, Katherine. A.; URBACH, Thomas P.; KUTAS, Marta. Probabilistic word pre-activation during language comprehension inferred from electrical brain activity. Nature Neuroscience, v. 8, n. 8, p. 1117-1121, ago. 2005.

DELONG, Katherine; URBACH, Thomas.; GROPPE, D.; KUTAS, Marta. Overlapping dual ERP responses to low cloze probability sentence continuations. Psychophysiology, v. 48, n. 9, p. 1203-1207, set. 2011.

DELONG, Katherine; QUANTE, L.; KUTAS, Marta., Predictability, plausibility and two late ERP positivities during written sentence comprehension, Neuropsychologia, v. 61, pp. 150-162, ago. 2014.

DIKKER, Suzanne; PYLKKÄNEN, Liina. Predicting language: MEG evidence for lexical preactivation. Brain \& Language, v. 1, n. 127, p. 55-64, out. 2013.

DIKKER, Suzanne; SILBERT, Lauren; HASSON, Uri; ZEVIN, Jason. Predictability enhances speakerlistener brain-to-brain synchrony in posterior superior temporal gyrus. The Journal of Neuroscience, v. 18, n. 34, p. 6267-6272, abr. 2014.

FARMER, Thomas. A.; BROWN, Meredith; TANENHAUS, Michael. K. Prediction, explanation, and the role of generative models in language processing [Commentary article]. Behavioral and Brain Sciences, v. 36, p. 211-212, jun. 2013.

FEDERMEIER, Kara D.; KUTAS, Marta. A rose by any other name: long-term memory structure and sentence processing. Journal of Memory and Language, v. 41, n. 9, p. 469-495, nov. 1999.

FEDERMEIER, Kara D.; KUTAS, Marta. Aging in context: age-related changes in context use during language comprehension. Psychophysiology, n. 42, v. 2, p. 133-141, mar. 2005.

FEDERMEIER, Kara D.; WLOTKO, Edward; OCHOADEWALD, Esmeralda de.; KUTAS, Marta. Multiple effects of sentential constraint on word processing. Brain Research, v. 1146, p. 75-84, mai. 2007.

FEDERMEIER, Kara D.; KUTAS, Marta; SCHUL, Rina. Age-related and individual differences in the use of prediction during language comprehension. Brain and Language, n. 115, v. 3, p. 149-161, dez. 2010.

FINE, Alex B.; JAEGER, T. Florian, Evidence for implicit learning in syntactic comprehension. Cognitive Science, v. 37, n. 3, p. 578-591, jan. 2013.

FODOR, Jerry A. The modularity of mind. Meaning and cognitive structures: Issues in the computational theory of mind, 1986, p. 3-18.

FORSTER, Kenneth. Basic issues in lexical 
processing. Lexical representation and process, ed. by W. Marslen-Wilson, 75-107. Cambridge, MA: The MIT Press, 1989.

FRAZIER, Lyn; FODOR, Janet D. The Sausage Machine: A new two-stage parsing model. Cognition, v. 6, n.4, p. 291-325, dez. 1978.

GRUTER, Theres; ROHDE, Hannah; SCHAFER, Amy. The Role of Discourse-Level Expectations in NonNative Speakers' Referential Choices. Proceedings of the 38th Annual Boston University Conference on Language Development (BUCLD). Somerville, MA: Cascadilla Press, 2014.

HAGOORT, Peter. The memory, unification, and control (MUC) model of language. In: MEYER, Antje S.; WHEELDON, Linda; KROTT, Andrea (Org.), Automaticity and control in language processing. Hove: Psychology Press, 2007, 287 p., p.243-270.

HINTZ, Florian; MEYER, Antje; HUETTIG, Falk. Event knowledge and word association jointly influence predictive processing during discourse comprehension. In: $28^{\text {th }}$ CUNY Conference on Human Sentence Processing, 2015, Los Angeles. Anais..., 2015, p. 175.

HUETTIG, Falk. Four central questions about prediction in language processing. Brain Research, n. 1626, p. 118-135, nov. 2015.

HUETTIG, Falk; BROUWER, Susanne. Delayed anticipatory spoken language processing in adults with dyslexia - Evidence from eye-tracking. Dyslexia, v. 21, n. 2, p 97-122, mai. 2015.

HUETTIG, Falk; JANSE, Esther. Individual differences in working memory and processing speed predict anticipatory spoken language processing in the visual world. Language, Cognition and Neuroscience, v. 1, n. 31, p. 80-93, jan. 2016.

HUETTIG, Falk; MANI, Nivedita. Is prediction necessary to understand language? Probably not. Language, Cognition and Neuroscience, v. 1, n. 31, p. 19-31, jan. 2016.

JACKENDOFF, Ray. Foundations of language: Brain, meaning, grammar, evolution. New York, NY: Oxford University Press, 2002, 504 p.

KAMIDE, Yuki; ALTMANN, Gerry TM; HAYWOOD, Sarah L. The time-course of prediction in incremental sentence processing: Evidence from anticipatory eye movements. Journal of Memory and language, v. 49, n. 1, p. 133-156, jul. 2003.

KEHLER, Andrew; ROHDE, Hannah. A Probabilistic Reconciliation of Coherence-Driven and CenteringDriven Theories of Pronoun Interpretation. Theoretical Linguistics, v. 39, n. 1-2, p. 1-37, set. 2013.

KIM, Christina S.; GUNLOGSON, Christine; TANENHAUS, Michael K.; RUNNER, Jeffrey T.
Context-driven expectations about focus alternatives. Cognition v. 139, p. 28-49, jun. 2015.

KUPERBERG, Gina R. Neural mechanisms of language comprehension: Challenges to syntax. Brain Research, v. 1146, n. 142, p. 23-49, mai. 2007.

KUPERBERG, Gina R. Separate streams or probabilistic inference? What the N400 can tell us about the comprehension of events. Language, Cognition and Neuroscience, p. 1-15, jan. 2016.

KUPERBERG, Gina. R.; JAEGER, T. Florian. What do we mean by prediction in language comprehension?, Language, Cognition and Neuroscience, v.31, n. 1, p. 32-50, nov. 2015.

KUTAS, Marta.; HILLYARD, Steve A. Reading senseless sentences: Brain potentials reflect semantic incongruity. Science, v. 207, n. 4427, p. 203-205, jan. 1980.

KUTAS, Marta; HILLYARD, Steven A. Brain potentials during reading reflect word expectancy and semantic association. Nature, v. 307, n. 5947, p. 1161-1163, jan. 1984.

KUTAS, Marta. In the company of other words: Electrophysiological evidence for single-word and sentence context effects. Language and cognitive processes, v. 8, n. 4, p. 533-572, 1993.

KUTAS, Marta; VAN PETTEN, Cyma K; KLUENDER, Robert. Psycholinguistics Electrified II (1994-2005). In: GERNSBACHER, Morton A.; TRAXLER, Matthew. (org.) Handbook of Psycholinguistics. 2nd edition. New York: Elsevier Press, 2006, p. 659724, $1184 \mathrm{p}$.

LASZLO, Sarah; FEDERMEIER, Kara D. A beautiful day in the neighborhood: An event-related potential study of lexical relationships and prediction in context. Journal of Memory and Language, v. 61, n. 3, p. 326-338, out. 2009.

LUCK, Steven. J. An Introduction to the Event-Related Potential Technique, second edition. Cambridge: MIT Press, 2014, 406 p.

MISHRA, Ramesh K.; SINGH, Niharika; PANDEY, Aparna; HUETTIG, Falk. Spoken languagemediated anticipatory eye movements are modulated by reading ability: evidence from Indian low and high literates. Journal of Eye Movement Research, n. 5, v. 1, p. 1-10, 2012.

OTTEN, Marte; VAN BERKUM, Jos JA. Discoursebased word anticipation during language processing: Prediction or priming?. Discourse Processes, v. 45, n. 6, p. 464-496, dez. 2008.

PICKERING, Martin. J.; GARROD, Simon. An integrated theory of language production and comprehension. Behavioral and Brain Sciences, v. 36, n. 04, p. 329-347, ago. 2013. 
ROHDE, Hannah; HORTON, William S. Anticipatory looks reveal expectations about discourse relations. Cognition, v. 133, n. 3, p. 667-691, dez. 2014.

SAFFRAN, Jenny; ASLIN, Richard; NEWPORT, Elissa. Statistical learning by 8 -month-old infants. Science, v. 274, n. 5294, p. 1926-1928, dez. 1996.

SALVERDA, Anne Pier; KLEINSCHMIDT, Dave; TANENHAUS, Michael K. Immediate effects of anticipatory coarticulation in spoken-word recognition. Journal of Memory and Cognition, v. 1, n. 71, p. 145-163, jan. 2014.

SMITH, Nathaniel. J.; LEVY, Roger. The effect of word predictability on reading time is logarithmic. Cognition, v. 128, n. 3, p. 302-319, set. 2013.

SCHWANENFLUGEL, Paula J.; SHOBEN, Edward J. The influence of sentence constraint on the scope of facilitation for upcoming words. Journal of Memory and Language, v. 24, n. 2, p. 232-252, abr. 1985.

SPIVEY-KNOWLTON, Michael J.; SEDIVY, Julie C. Resolving attachment ambiguities with multiple constraints. Cognition, n. 55, v. 3, p. 227-267, jun. 1995.

TAYLOR, Wilson L. Cloze procedure: a new tool for measuring readability. Journalism Quarterly, v. 30, n. 4, p. 415-433, set. 1953.

THORNHILL, DIANNE E.; VAN PETTEN, CYMA. Lexical versus conceptual anticipation during sentence processing: Frontal positivity and N400 ERP components. International Journal of Psychophysiology, v. 83, n. 3, p. 382-392, mar. 2012.

TRAXLER, Matthew J.; FOSS, Donald J. Effects of sentence constraint on priming in natural language comprehension. Journal of Experimental Psychology: Learning, Memory, and Cognition, v. 26, n. 5, p. 1266- 1282, set. 2000.

VAN BERKUM, Jos J. A; BROWN, Colin M.; ZWITSERLOOD, Pienie; KOOIJMAN, Valesca;
HAGOORT, Peter. Anticipating upcoming words in discourse: evidence from ERPs and reading times. Journal of Experimental Psychology: Learning, Memory, and Cognition, v. 31, n. 3, p. 443, mai. 2005.

VAN PETTEN, Cyma; KUTAS, Marta. Interactions between sentence context and word frequency in event-related brain potentials. Memory \& Cognition, v. 18 , n. 4 , p. 380-393, mai. 1990.

VAN PETTEN, Cyma; LUKA, Barbara J. Prediction during language comprehension: Benefits, costs, and ERP components. International Journal of Psychophysiology, v. 83, n. 2, p. 176-190, fev. 2012.

VIEIRA, Cláudia. B. A atuação de conhecimentos de eventos na compreensão da leitura. 2015. $104 \mathrm{f}$. Dissertação (Mestrado em Linguística). Curso de Pós Graduação em Estudos Linguísticos, Faculdade de Letras, Universidade Federal de Minas Gerais, Belo Horizonte, 2015.

WICHA, Nicole Y.; MORENO, Eva M.; KUTAS, Marta. Expecting gender: An event related brain potential study on the role of grammatical gender in comprehending a line drawing within a written sentence in Spanish. Cortex, v. 39, n. 3, p. 483-508, jun. 2003 .

WICHA, Nicole Y.; MORENO, Eva M.; KUTAS, Marta. Anticipating words and their gender: an event related brain potential study of semantic integration, gender expectancy, and gender agreement in Spanish sentence reading. Journal of Cognitive Neuroscience, v.16, n.7, p.1272-1288, set. 2004.

WLOTKO, Edward; FEDERMEIER, Kara D. Agerelated changes in the impact of contextual strength on multiple aspects of sentence comprehension. Psychophysiology, v. 49, n. 6, p. 770-785, jun. 2012.

WLOTKO, Edward W.; FEDERMEIER, Kara D. Time for prediction? The effect of presentation rate on predictive sentence comprehension during wordby-word reading. Cortex, v. 68, p. 20-32, jul. 2015.

\section{COMO CITAR ESSE ARTIGO}

GODOY, Mahayana Cristina; VIEIRA, Cláudia Brandão; CUNHA LIMA, Maria Luiza. Antecipação e processamento linguístico: uma revisão das principais evidências a favor da previsão. Signo, Santa Cruz do Sul, v. $41, \quad$ n. $71, \quad$ set. 2016. ISSN 1982-2014. Disponível em: $<$ https://online.unisc.br/seer/index.php/signo/article/view/7227>. Acesso em: doi: http://dx.doi.org/10.17058/signo.v41i71.7227. 